\title{
A Miniature Jumping Robot with Self-Recovery Capabilities
}

\author{
Mirko Kovač, Manuel Schlegel, Jean-Christophe Zufferey and Dario Floreano
}

\begin{abstract}
In nature, many animals are able to jump, upright themselves after landing and jump again. This allows them to move in unstructured and rough terrain. As a further development of our previously presented $7 \mathrm{~g}$ jumping robot, we consider various mechanisms enabling it to recover and upright after landing and jump again. After a weighted evaluation of these different solutions, we present a spherical system with a mass of $9.8 \mathrm{~g}$ and a diameter of $12 \mathrm{~cm}$ that is able to jump, upright itself after landing and jump again. In order to do so autonomously, it has a control unit and sensors to detect its orientation and spring charging state. With its current configuration it can overcome obstacles of $76 \mathrm{~cm}$ at a take-off angle of $75^{\circ}$.
\end{abstract}

\section{INTRODUCTION}

Locomotion in rough and unstructured terrain is naturally very difficult for small animals or robots [1]. One efficient solution is to perform repetitive jumping in order to be able to overcome relatively large obstacles and travel at a low energetic cost. In nature, many animals such as locusts [2], springtails [3], click beatles [4] and fleas [5] use jumping as their principal means of locomotion. However, a challenge that they inevitably have to face is to be able to upright themselves after landing in order to jump again. To achieve this, they use a variety of mechanisms and behaviors [6][7].

In robotics, various jumping systems have been presented so far using different approaches for jumping [8][11]-[18], but only very few are capable of uprighting themselves after landing. To the best of our knowledge, none has been shown so far that is at the same range of mass and jumping performance as the system presented in this paper (see table I for an overview on similar existing jumping robots).

Our previously presented miniature jumping robot has established a record at its size and weight. With a mass of $7 \mathrm{~g}$ at a size of $5 \mathrm{~cm}$ [8][9] it can clear heights of up to $1.4 \mathrm{~m}$. However, its major limitation was its inability to upright itself after landing.

As a further development of this robot, we present in the following sections a spherical system of less than ten grams (figure 1) that is able to jump, upright itself after landing and jump again. Due to the spherical structure of our solution, it is also able to perform passive rolling, similar to rolling animals, such as the Namib Golden Wheel spider or the Web-toed salamander [10]. We start by outlining the conceptual design and evaluation phase, the integration

This project is funded by EPFL and by the Swiss National Science Foundation, grant number 200021-105545/1

All authors are with the Ecole Polytechnique Fédérale de Lausanne (EPFL), Laboratory of Intelligent Systems (LIS, http://lis.epfl.ch), CH-1015 Lausanne, Switzerland mirko.kovac, manuel.schlegel, jean-christophe.zufferey, dario.floreanodepfl.ch

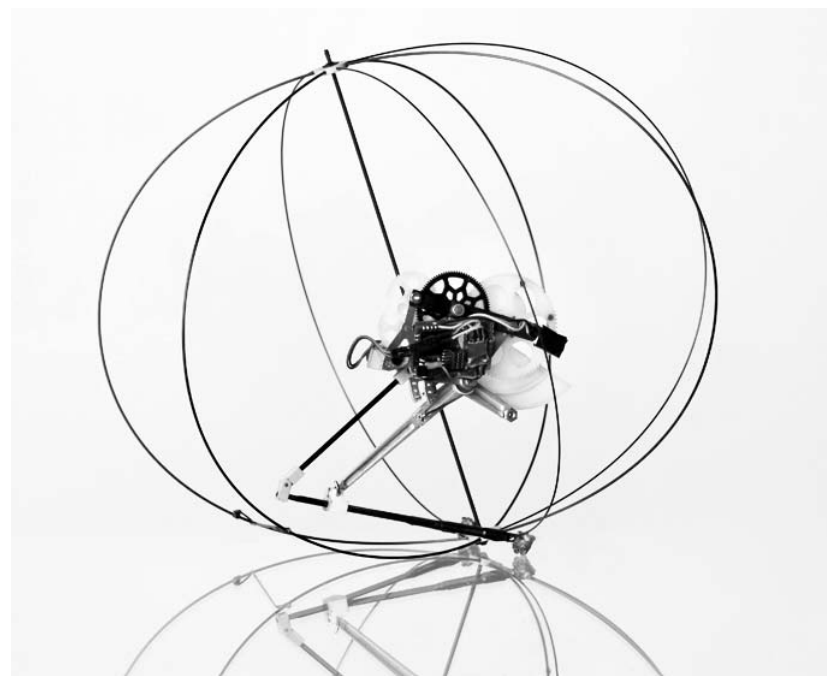

Fig. 1. 9.8g jumping robot able to jump, roll passively, upright itself after landing and jump again. The cage has a diameter of $12 \mathrm{~cm}$.

of the chosen solution with the existing jumping robot, then we give an overview on its electronics and control and we finally characterize the performance of our working and autonomous prototype. High-speed video footage of its behavior can be seen in the accompanying material and at http://lis.epfl.ch/jumpglider.

\section{DESIGN}

\section{A. Jumping mechanism}

The main design requirement for the jumping mechanism is to be a lightweight propulsion unit for jumping robots, with the possibility to adjust the jumping height and takeoff angle. It has been shown that for small jumping systems the highest jumps can be achieved by first slowly charging an elastic element and then using the legs as catapult to jump [8][18][19][20]. This jumping principle is used by small animals such as desert locusts [2], fleas [5] and frogs [21] to perform very powerful jumps. The working principle in the design used for our jumping robot is to first charge a torsion spring and then quickly release its energy to extend a four bar leg linkage to jump, as illustrated in figure 2. Our previously presented minimalist jumping robot [8] uses successfully the exact same principles. The basic components of the design are the four bar leg mechanism that is connected to the body on the ground link (a) and is actuated via the input link (b) using a torsion spring (c).

The mechanical implementation (figure 3) of this basic working principle is realized by using a $4 \mathrm{~mm}$ DC motor 
TABLE I

STATE OF THE ART

\begin{tabular}{|c|c|c|c|c|c|}
\hline Name & mass $[\mathrm{g}]$ & approx. jump height $[\mathrm{cm}]$ & jump height per mass $[\mathrm{cm} / \mathrm{g}]$ & uprighting & passive rolling \\
\hline Sandia robot [11] & 2500 & 300 & 0.12 & yes & no \\
\hline Rescue robot [12] & 2000 & 80 & 0.04 & yes & yes \\
\hline Minimalist jumping robot [13] & 1300 & 90 & 0.07 & yes & no \\
\hline Jollbot [14] & 465 & 21.8 & 0.05 & yes & yes \\
\hline Spherical crawling/rolling robot [15] & N/A & 39 & N/A & no & yes \\
\hline Scout [16] & 200 & 35 & 0.18 & yes & no \\
\hline Mini-Whegs [17] & 190 & 22 & 0.12 & no & no \\
\hline Grillo [18] & $8-80$ & 5 & $0.63-0.06$ & no & no \\
\hline EPFL jumping robot [8] & 7 & 138 & 19.7 & no & no \\
\hline Jumping robot presented here & 9.8 & 76 & 7.76 & yes & yes \\
\hline
\end{tabular}

Ready to jump
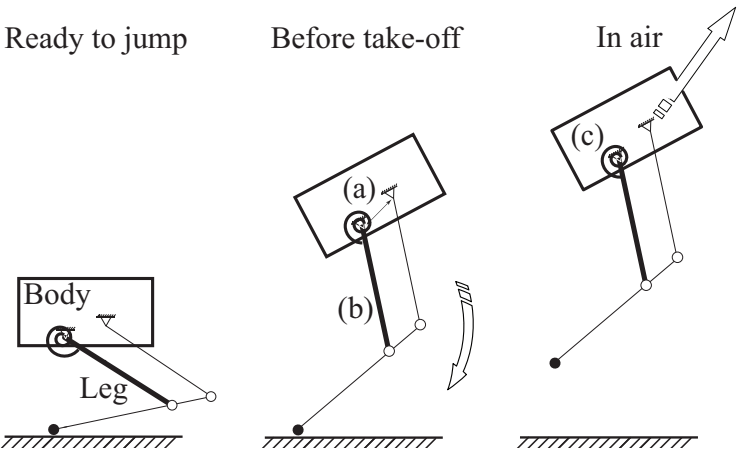

Fig. 2. Working principle for the jumping mechanism. To jump, a four bar leg linkage, which is connected to the body on the ground link (a) is extended via the input link (b) using a torsion spring (c).

(a) to turn a cam (b) by way of a four stage gear box (c). The motor turns the cam in counterclockwise direction in order to charge two torsion springs (d). These two springs are located around the axis of the leg (e) and are fixed to the frame (f) and the main leg (g). Once the outermost point of the cam is reached, the energy that is stored in the springs actuates the main leg which is the input link for the four bar leg mechanism. By changing the spring setting (h) and the geometry of the legs, the jumping height, take-off angle and ground force profile can be adjusted [22]. A jump can be performed every $3 \mathrm{~s}$ at a power consumption of $350 \mathrm{~mW}$. The interested reader may refer to [8] for a more detailed explanation and characterization of the jumping principles used.

\section{B. Uprighting mechanism}

The qualitative requirements of the uprighting mechanism are (i) very low weight, (ii) simple construction, (iii) mechanical robustness and (iv) low power consumption in performing the uprighting movement. As a first step in our development process we considered four different designs regarding the shape of the uprighting mechanism and its integration with the existing jumping robot (figure 4).

In solution A, a cage consisting of an upper part (a) and a lower part (b) is attached to (a) the body and (b) the tip of the

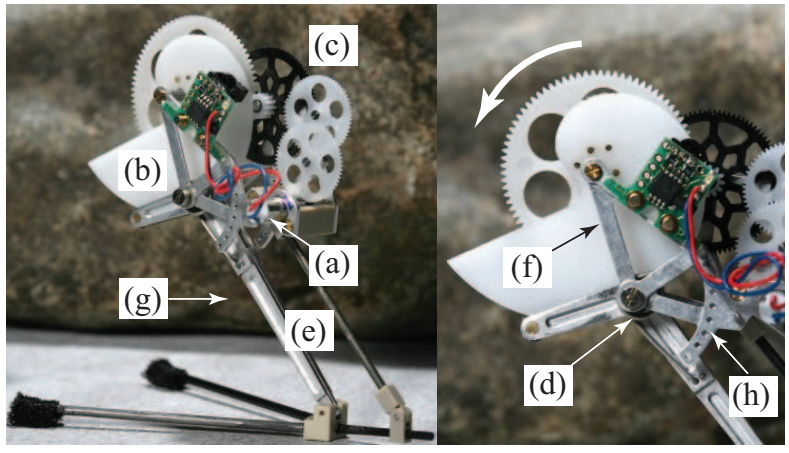

Fig. 3. Jumping mechanism that presents the propulsion unit for our robot (a) $4 \mathrm{~mm}$ DC pager motor, (b) cam, (c) four stage gear box, (d) two steel torsion springs, (e) four bar linkage leg structure, (f) aluminum frame, (g) main leg as input link, (h) spring setting.

foot of the jumping robot. As the jumping robot charges for the next jump, it contracts the legs and naturally the center of gravity of the entire structure is moved towards the lower part, which leads to a passive uprighting movement. In order to be able to contract the cage as the robot charges for the next jump, the rods from the lower part slide along the rods of the upper part, which inevitable produces kinetic friction and calls for an increase of complexity to ensure its structural robustness.

Solution B consists of a spherical cage which is attached to the body and the feet of the jumping robot. Right after landing, the robot will rest in its stable position which is upside down. As soon as the jumping robot charges for the next jump, the cage is squeezed to an ellipsoid and the center of gravity moves to the lower part of the structure. One potential drawback of this solution is that if the uprighting movement is obstructed, there is a certain risk that the robot may end up in its second stable position, which is upside down. In addition, this solution has a higher associated energy cost due to the squeezing of the structure.

The third possibility that we considered, solution $\mathrm{C}$, consists of a stable spherical cage that is attached to the feet of the jumping robot. When charging for the next jump, the body slides down a rail (c) and shifts the center of 
A: After landing Ready for take-off

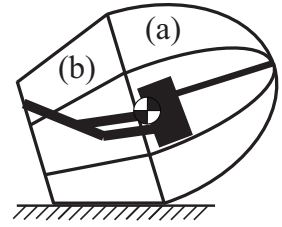

$\mathrm{B}$ :
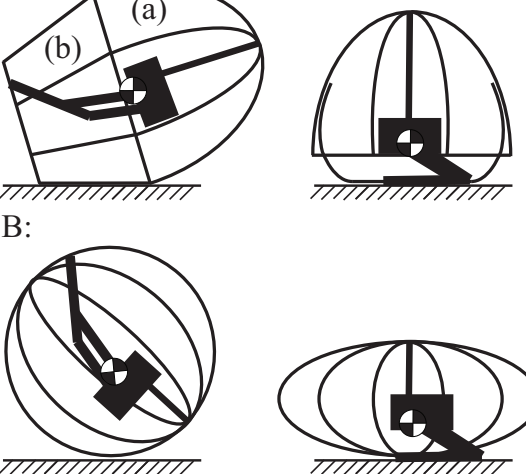

$\mathrm{C}$ :

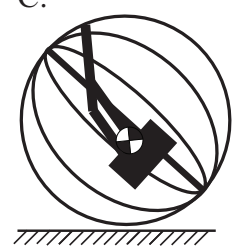

D:
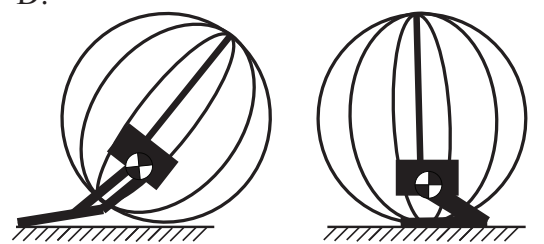

(c)

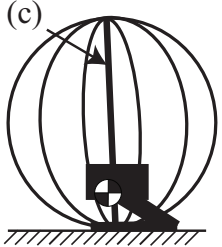

Center of gravity

Fig. 4. Four possible solutions for the mechanical design of the uprighting mechanism. (a) upper part of the cage and (b) lower part of the cage in solution A. (c) rail for solution C.
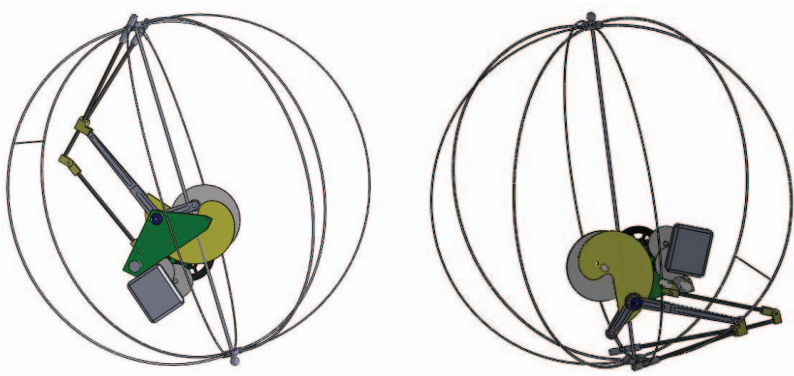

Fig. 5. Chosen solution $\mathrm{C}$ in $\mathrm{CAD}$.

gravity of the entire structure to the lower part of the sphere which leads to the desired uprighting movement. Since there is no deformation performed on the cage, this solution is structurally simpler, more energy efficient, and the load on the components is lower than in the first two solutions, which increases its mechanical robustness.

The fourth and final solution D, is similar to solution C, only that the cage is attached to the body and not to the feet of the robot. The shortcoming of this solution however is

TABLE II

WEIGHTED EVALUATION OF THE FOUR DIFFERENT CONCEPTUAL DESIGNS FOR THE UPRIGHTING MECHANISM

\begin{tabular}{|c|c||c|c||c||c|}
\hline Criteria & Weight & (A) & (B) & (C) & (D) \\
\hline Weight & 0.4 & 2 & 4 & $\mathbf{3}$ & 4 \\
\hline Simplicity & 0.1 & 1 & 3 & $\mathbf{5}$ & 3 \\
\hline Robustness & 0.2 & 2 & 3 & $\mathbf{5}$ & 3 \\
\hline Energy consumption & 0.3 & 3 & 1 & $\mathbf{5}$ & 5 \\
\hline \hline Total & 1 & 2.2 & 2.8 & $\mathbf{4 . 2}$ & 4 \\
\hline
\end{tabular}

(1: very unfavorable - 5: very favorable)

that the legs are outside the cage on landing which exposes them to potential damage.

In order to compare these four possible solutions for the design of the uprighting mechanism, we perform a weighted comparative evaluation [23] (see the evaluation matrix in table II) and decide on implementing solution $\mathrm{C}$ due to its structural simplicity, robustness and the additional benefit of being able to roll passively, which may be difficult with the other designs.

\section{Mechanical and electronic design}

We implemented the chosen solution in SolidWorks (figure 5), a Computer Aided Design (CAD) program and assemble the cage using commercially available carbon rods and connecting parts that are produced using a 3D printer [24] out of ABSplus, a light-weight and relatively strong thermoplastic material. The design details of our robot are illustrated in figure 6 . The structure of the cage consists of the $1 \mathrm{~mm}$ carbon rail (a) and $0.7 \mathrm{~mm}$ carbon rods that are held together by the connection piece (b). The printed circuit board (c) is populated with a Microchip Pic18LF4620 microcontroller, a Freescale MMA7260 three-axis accelerometer and an $\mathrm{H}$ bridge motor driver, and is powered using a Full River $10 \mathrm{mAh}$ Lithium Polymer battery. The detection of the charging state is done using a hall sensor (e) and two small magnets (d) that are integrated on the last gear stage. The uprighting mechanism is fixed on the jumping robot at the feet (f). During the acceleration phase before take-off, the body of the robot slides along the rail and the tips of the feet stick out of the cage to ensure contact with the ground.

\section{Control strategy}

The control strategy of our robot is illustrated in figure 7. Using the electronics mentioned above, the robot is able to detect its orientation and charging state. As soon as it is upright, it will jump autonomously. The in-air position is defined as the overall acceleration being less than $0.7 \mathrm{~m} / \mathrm{s}^{2}$. Once in-air, it charges only partially to keep the center of gravity of the entire robot close to the middle of the cage and to facilitate subsequent rolling. After landing and settling down (acceleration values constant over a period of 500ms), it charges itself completely in order to shift its center of 


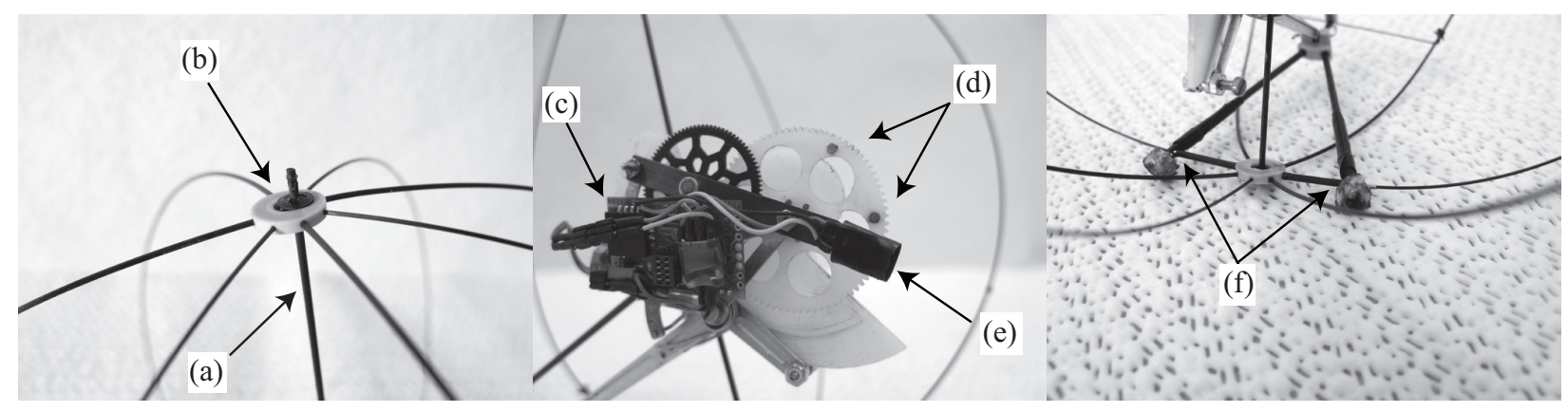

Fig. 6. Mechanical design details and integration with the jumping system. (a) rail, (b) connection piece, (c) printed circuit board and electronics, (d) two magnets on the last gear stage, (e) hall sensor, (f) integration of the cage with the feet.

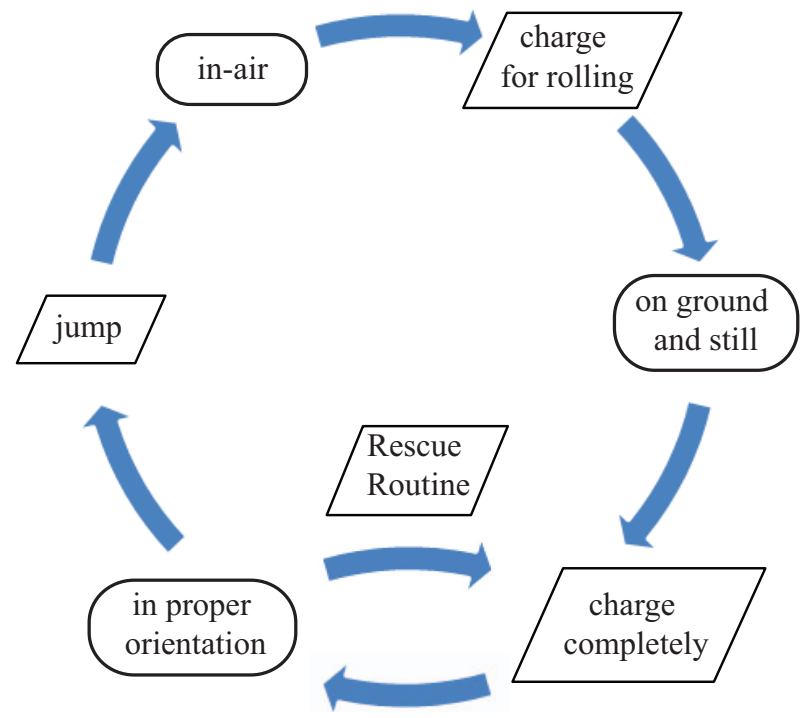

Fig. 7. Overview of the control strategy, represented as flowchart.

gravity to the bottom part of the cage, thus uprights itself and jumps again. If the orientation is still not upright after charging and just before the jump, it uses a so called 'rescue routine' (RR) to try again to upright itself. The RR consists of a discharging and re-charging cycle. If this $\mathrm{RR}$ is performed three times consecutively and the position is still not upright, the robot kicks by releasing a jump in order to free itself from potential stuck situations and starts again with charging completely (see the characterization of the RR in section IIIC).

\section{RESUlTS}

\section{A. Uprighting and charging}

The prototype as described above has a diameter of $12 \mathrm{~cm}$ and weights $9.8 \mathrm{~g}$ all together, including battery and electronics (weight budget in table III). A complete uprighting and take-off sequence is illustrated in figure 8. The durations, velocities and following trajectories are measured optically, using a high-speed camera system [25] at 500 frames per
TABLE III

WEIGHT BUDGET

\begin{tabular}{|c|c|}
\hline Part & Mass $[\mathrm{g}]$ \\
\hline \hline Total mass of the jumping mechanism & 6.87 \\
\hline \hline Carbon cage & 0.85 \\
\hline Carbon rail & 0.14 \\
\hline Electronics & 1.31 \\
\hline LiPo Battery 10mAh & 0.63 \\
\hline \hline Total mass of the complete prototype & $\mathbf{9 . 8 0}$ \\
\hline
\end{tabular}

second and adequate motion analysis software [26]. The charging cycle takes $3 \mathrm{~s}$ whereby the uprighting movement happens in $0.7 \mathrm{~s}$ in case the robot is not obstructed. Once jumping, the take-off velocity of $3.52 \mathrm{~m} / \mathrm{s}$ is reached in $17 \mathrm{~ms}$.

\section{B. Jumping performance}

The trajectory of the robot jumping out of a box of $50 \mathrm{~cm}$ depth is shown in figure 9. At a take-off angle of $75^{\circ}$ it reaches a jump height of $76 \mathrm{~cm}$, with a horizontal velocity at the top of the trajectory of $0.93 \mathrm{~m} / \mathrm{s}$. The comparison of the system with and without uprighting mechanism can be seen in figure 10. The jumping height of the system without uprighting mechanism is $103.3 \mathrm{~cm}$ at a take-off velocity of $3.92 \mathrm{~m} / \mathrm{s}$. The height loss due to the addition of the uprighting ability is $27.3 \mathrm{~cm}$ which corresponds to $26.4 \%$ compared to the system without the uprighting mechanism. This height loss is due to the mass increase of $2.8 \mathrm{~g}$ and the fact that the cage experiences oscillations right after take-off (see movie in the accompanying material). These oscillations are lost energy that cannot be converted into jumping height, as described by Alexander et al. for jumping systems with 'heavy feet' [4]. The jumping performance can thus be further improved by reducing the weight of the components and increasing the rigidity of the carbon cage structure.

\section{Rescue routine}

There are different situations to challenge the uprighting movement by testing the robot in obstructed situations. One scenario where this is the case is when the robot is stuck 


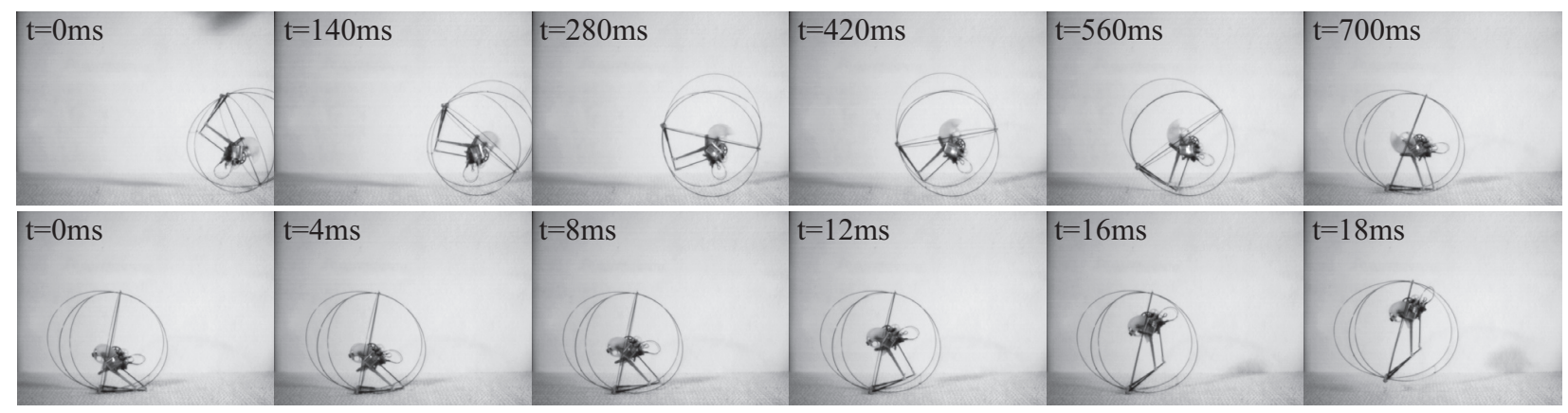

Fig. $8.1^{\text {st }}$ row: The uprighting movement takes $700 \mathrm{~ms} .2^{\text {nd }}$ row: The take-off velocity of $3.52 \mathrm{~m} / \mathrm{s}$ is reached in $17 \mathrm{~ms}$.

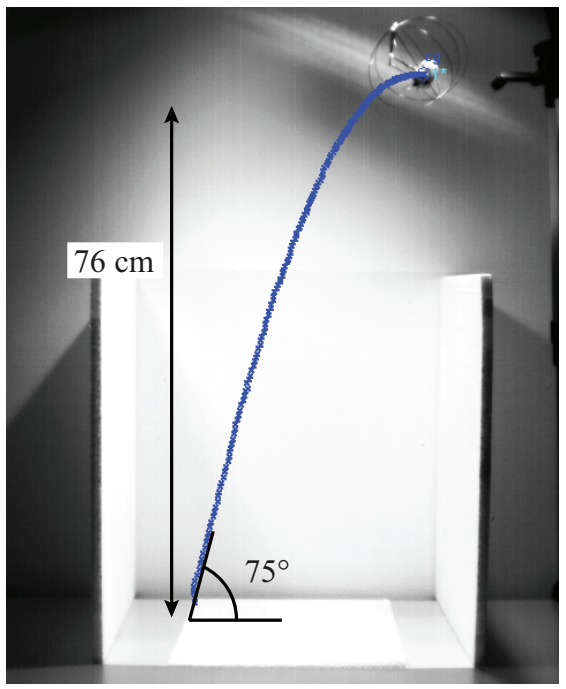

Fig. 9. Jumping trajectory of our robot, jumping out of a box of $50 \mathrm{~cm}$ depth. The jumping height is $76 \mathrm{~cm}$ at a take-off angle of $75^{\circ}$.

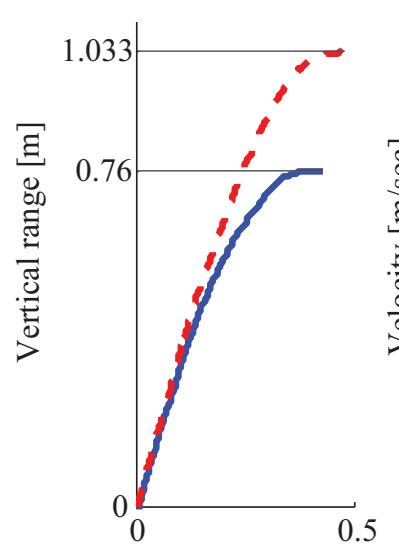

Horizontal range $[\mathrm{m}]$
Horizontal range $[\mathrm{m}]$

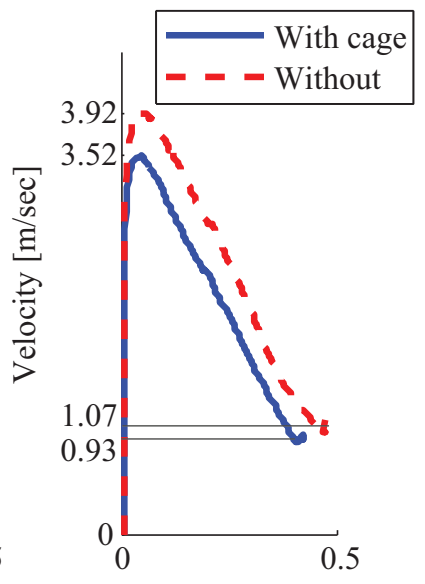

Fig. 10. Comparison of the trajectories and velocity profiles of the jumping robot with the uprighting cage and without. At a take-off angle of $75^{\circ}$, the caged system is able to jump to a height of $76 \mathrm{~cm}$, whereas the cage-less system reaches a height of $103.3 \mathrm{~cm}$. The velocity profiles are qualitatively similar, the take-off velocities are $3.92 \mathrm{~m} / \mathrm{s}$ and $3.52 \mathrm{~m} / \mathrm{s}$ respectively.

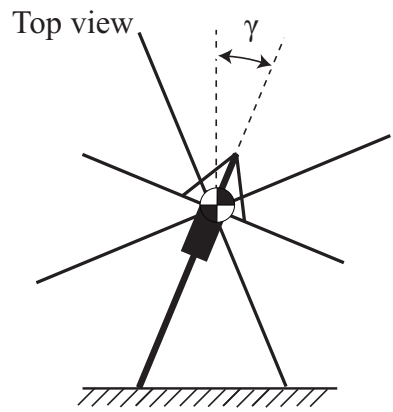

Side view

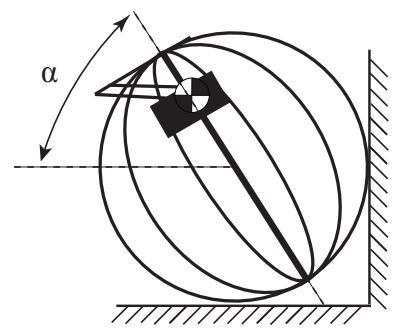

Fig. 11. We put the robot in 35 different positions facing a vertical wall and measure the time the robot needs to get away from the wall and perform a regular jump at a take-off angle of $75^{\circ}$.

in a corner of the ground and a vertical wall (figure 11). As an attempt to systematically characterize its ability to free itself from such stuck situations, we place the robot in five different pitch angles $\left(\alpha=0^{\circ}, 45^{\circ}, 90^{\circ}, 135^{\circ}, 180^{\circ}\right)$ and for every one of them in seven yaw angles $\left(\gamma=0^{\circ}, 55^{\circ}, 112.5^{\circ}, 157.5^{\circ}, 202.5^{\circ}, 247.5^{\circ}, 305^{\circ}\right)$ facing the wall. We perform one jump for every of the 35 combinations of pitch and jaw and observe the behavior (table IV). To characterize which position is the most difficult for the robot to free itself from, we measure the time in this set of 35 jumps that the robot needs until it can perform a regular jump at a take-off angle of $75^{\circ}$ (figure 12 ). The data shows that it successfully uses the RR and manages to get out of the situation in every case, whereby the most difficult position is when it is stuck upside down, facing the wall on its back $\left(\gamma=0^{\circ}\right.$ and $\left.\alpha=180^{\circ}\right)$.

\section{CONCLUSION}

We present a $12 \mathrm{~cm}, 9.8 \mathrm{~g}$ jumping robot that is able to jump, upright itself after landing, detect its orientation and jump again within 3s. A control unit and sensors, allow the robot to do this autonomously. In addition, it is able to roll passively and can free itself from stuck situations where it is facing a vertical wall. The jumping height of the current configuration is $76 \mathrm{~cm}$ at a take-off angle of $75^{\circ}$, which is $27.3 \mathrm{~cm}$ lower compared to the same robot without the spherical uprighting mechanism. To adjust the jump- 
TABLE IV

USE OF THE 'RESCUE ROUTINE' (RR) BY THE ROBOT TO FREE ITSELF FACING A VERTICAL WALL AT THE PITCH ANGLE $\alpha$ AND THE YAW ANGLE $\gamma$

\begin{tabular}{|c|c|c|c|c|c|c|c|}
\hline & $\gamma=0^{\circ}$ & $\gamma=55^{\circ}$ & $\gamma=112.5^{\circ}$ & $\gamma=157.5^{\circ}$ & $\gamma=202.5^{\circ}$ & $\gamma=247.5^{\circ}$ & $\gamma=305^{\circ}$ \\
\hline$\alpha=0^{\circ}$ & 0 & 0 & 0 & 0 & 0 & 0 & 0 \\
\hline$\alpha=45^{\circ}$ & $1 \mathrm{rr}$ & $2 \mathrm{rr}$ & $1 \mathrm{rr}$ & $3 \mathrm{rr}+\mathrm{k}$ & $3 \mathrm{rr}+\mathrm{k}$ & $3 \mathrm{rr}+\mathrm{k}$ & $1 \mathrm{rr}$ \\
\hline$\alpha=90^{\circ}$ & $3 \mathrm{rr}+\mathrm{k}$ & $1 \mathrm{rr}$ & $1 \mathrm{rr}$ & $1 \mathrm{rr}$ & $1 \mathrm{rr}$ & $1 \mathrm{rr}$ & $1 \mathrm{rr}$ \\
\hline$\alpha=135^{\circ}$ & $3 \mathrm{rr}+\mathrm{k}$ & $3 \mathrm{rr}$ & 0 & 0 & 0 & 0 & $1 \mathrm{rr}$ \\
\hline$\alpha=180^{\circ}$ & $3 \mathrm{rr}+\mathrm{k}+3 \mathrm{rr}+\mathrm{k}$ & $3 \mathrm{rr}+\mathrm{k}+3 \mathrm{rr}+\mathrm{k}$ & $3 \mathrm{rr}+\mathrm{k}+1 \mathrm{rr}$ & $1 \mathrm{rr}$ & $3 \mathrm{rr}+\mathrm{k}$ & $1 \mathrm{rr}$ & $3 \mathrm{rr}+\mathrm{k}$ \\
\hline
\end{tabular}

(0: no need for RR, Nrr: execution of RR N times, k: kick

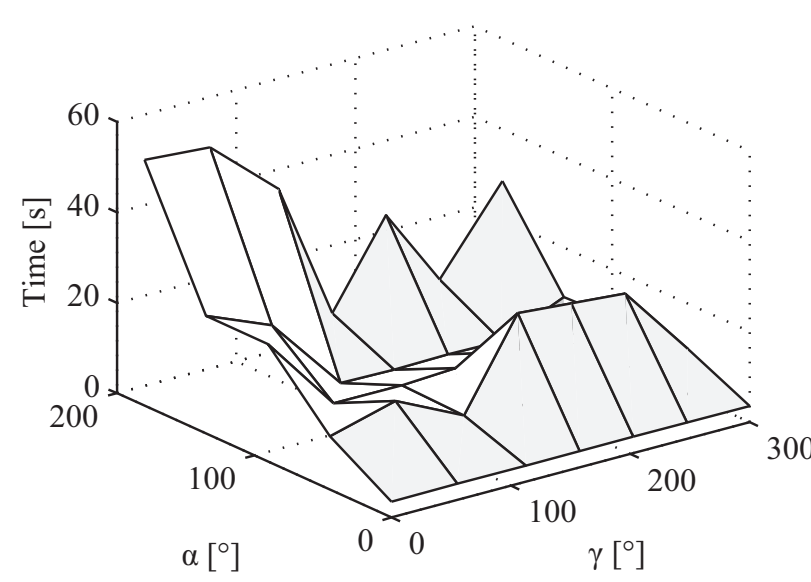

Fig. 12. Time the robot needs to get away from the wall ( $\alpha$ pitch and $\gamma$ yaw angle) and perform a regular jump at a take-off angle of $75^{\circ}$.

ing behavior to different environments, the take-off angle, jumping height and ground force profile can be adjusted. Future work addresses different ways that allow the robot to jump in a given direction and its integration with sensors and communication devices to perform tasks as a collective system.

\section{ACKNOWLEDGEMENT}

The authors would like to thank Walter Karlen for lending us his printed circuit board and for his support to set it up. We would also like to thank the Atelier de l'Institut de production et robotique (ATPR) at EPFL for the fabrication of the parts. This project is funded by EPFL and by the Swiss National Science Foundation, grant number 200021-105545/1.

\section{REFERENCES}

[1] M. Kaspari and M. D. Weiser, "The sizegrain hypothesis and interspecific scaling in ants," Functional Ecology, vol. 13, no. 4, pp. 530-538, 1999.

[2] H. C. Bennet-Clark, "The energetics of the jump of the locust Schistocerca gregaria," Journal of Experimental Biology, vol. 63, no. 1 , pp. 53-83, 1975.

[3] J. Brackenbury and H. Hunt, "Jumping in springtails: mechanism and dynamics," Journal of Zoology, vol. 229, pp. 217-236, 1993.

[4] R. M. Alexander, Principles of Animal Locomotion. Princeton University Press, 2003.

[5] W. Gronenberg, "Fast actions in small animals: springs and click mechanisms," Journal of Comparative Physiology A: Sensory, Neural, and Behavioral Physiology, vol. 178, no. 6, pp. 727-734, 1996.
[6] A. Faisal, "Coordinated righting behaviour in locusts," Journal of Experimental Biology, vol. 204, no. 4, pp. 637-648, 2001.

[7] L. Frantsevich, "Righting kinematics in beetles (insecta: Coleoptera)," Arthropod Structure and Development, vol. 33, no. 3, pp. 221-235, 2004.

[8] M. Kovac, M. Fuchs, A. Guignard, J. Zufferey, and D. Floreano, "A miniature $7 \mathrm{~g}$ jumping robot," in IEEE International Conference on Robotics and Automation, 2008, pp. 373-378.

[9] M. Kovac, J. Zufferey, and D. Floreano, "Towards a self-deploying and gliding robot," in Flying Insects and Robots, D. Floreano, J.-C. Zufferey, M. V. Srinivasan, and C. Ellington, Eds. Springer, 2009, ch. 19.

[10] R. H. Armour and J. F. V. Vincent, "Rolling in nature and robotics: A review," Journal of Bionic Engineering, vol. 3, no. 4, pp. 195-208, 2006.

[11] P. Weiss, "Hop... hop... hopbots!: designers of small, mobile robots take cues from grasshoppers and frogs," Science News, vol. 159, p. 88, 2001.

[12] H. Tsukagoshi, M. Sasaki, A. Kitagawa, and T. Tanaka, "Design of a higher jumping rescue robot with the optimized pneumatic drive," in IEEE International Conference on Robotics and Automation, 2005, pp. 1276-1283.

[13] J. Burdick and P. Fiorini, "Minimalist jumping robot for celestial exploration," The Internation Journal of Robotics Research, vol. 22, no. 7, pp. 653-674, 2003

[14] R. Armour, K. Paskins, A. Bowyer, J. F. V. Vincent, and W. Megill, "Jumping robots: a biomimetic solution to locomotion across rough terrain," Bioinspiratoin and Biomimetics Journal, vol. 2, pp. 65-82, 2007.

[15] Y. Sugiyama, M. Yamanaka, and S. Hirai, "Circular/spherical robots for crawling and jumping," in IEEE International Conference on Robotics and Automation, 2005, pp. 3595-3600.

[16] S. A. Stoeter, P. E. Rybski, and N. Papanikolopoulos, "Autonomous stair-hopping with scout robots," in IEEE/RSJ International Conference on Intelligent Robots and Systems, vol. 1, 2002, pp. 721-726.

[17] B. G. A. Lambrecht, A. D. Horchler, and R. D. Quinn, "A small, insectinspired robot that runs and jumps," in International Conference on Robotics and Automation, 2005, pp. 1240- 1245.

[18] U. Scarfogliero, C. Stefanini, and P. Dario, "Design and development of the long-jumping" grillo" mini robot," in IEEE International Conference on Robotics and Automation, 2007, pp. 467-472.

[19] R. M. Alexander, Elastic Mechanisms in Animal Movement. Cambridge University Press, 1988.

[20] M. Burrows, "Biomechanics: Froghopper insects leap to new heights," Nature, vol. 424, no. 6948, p. 509, 2003.

[21] T. J. Roberts and R. L. Marsh, "Probing the limits to musclepowered accelerations: lessons from jumping bullfrogs," Journal of Experimental Biology, vol. 206, no. 15, pp. 2567-2580, 2003.

[22] M. Kovac, J. Zufferey, and D. Floreano, "Towards the self deploying microglider, a biomimetic jumping and gliding robot," in Proceedings of the 4th International Symposium on Adaptive Motion of Animals and Machines, 2008, pp. 41-42.

[23] D. G. Ullman, The mechanical design process. McGraw-Hill, 2002.

[24] http://www.funtech.com. (2008) Dimension elite 3d printer.

[25] http://www.fastecimaging.com. (2007) Troubel shooter 1000 high speed camera.

[26] http://www.xcitex.com. (2008) Proanalyst motion analysis software. 